\title{
Is pharmacological treatment of pain harmful or beneficial?
}

Katsuhiro Toda*

Department of Orthopedic Surgery, Kitahiroshima Town Toyohira Hospital, 4705, Azaka, Kita-Hiroshima Town, Yamagata-Gun, Hiroshima, 731-1222, Japan

\begin{abstract}
If pharmacological treatment alleviates pain, it may cause delay in starting appropriate treatment. If a medicine is effective for lumbar canal stenosis, operation of lumbar canal stenosis is delayed. Cauda equine and/or lumbar nerve root is repeatedly damaged for a long time while the medicine alleviates pain and/or numbness. It may cause poor treatment outcome after operation. Pharmacological treatment often alleviates osteoarthritis, resulting in reduce motivation of weight reduction or rest. Physician and patient should know the dilemma in case that pharmacological treatment is performed.
\end{abstract}

Pain itself is an invasion such as infection. Based on the theory of central sensitization [1], all kinds of long-term pain cause central sensitization that is a responsible lesion of new pain. Therefore, pharmacological treatment of pain is beneficial even if it sometimes causes adverse effects.

Adverse effects due to pharmacological treatment are harmful. However, pharmacological treatment of pain itself is sometimes harmful. If pharmacological treatment alleviates pain, it may cause delay in starting appropriate treatment.

If a medicine is effective for lumbar canal stenosis, operation of lumbar canal stenosis is delayed. Cauda equine and/or lumbar nerve root is repeatedly damaged for a long time while the medicine alleviates pain and/or numbness. It may cause poor treatment outcome after operation. There is similar worry in trigeminal neuralgia [2]. A patient with obesity and osteoarthritis of the knee has to lose weight. A patient with overuse syndrome has to rest the affected area. Pharmacological treatment often alleviates such pain, resulting in reduce motivation of weight reduction or rest. Ultimately, it causes destruction of the living body, resulting in artificial joint replacement, stress fracture, etc. I am afraid that pharmacological treatment of pain may be harmful on this point. In such cases, we have to explain that effective pharmacological treatment may be harmful and weight reduction or rest are necessary. Physician and patient should know the dilemma in case that pharmacological treatment is performed.

\section{Authors declaration}

No conflicts of interest have been declared.

\section{References}

1. Woolf CJ (2011) Central sensitization: implications for the diagnosis and treatment of pain. Pain 152 (3 Suppl): S2-15.

2. Toda K (2008) Operative treatment of trigeminal neuralgia: review of current techniques. Oral Surg Oral Med Oral Pathol Oral Radiol Endod 106: 788-805, 805 e1-6. [Crossref]
Copyright: (C)2018 Toda K. This is an open-access article distributed under the terms of the Creative Commons Attribution License, which permits unrestricted use, distribution, and reproduction in any medium, provided the original author and source are credited.
${ }^{*}$ Correspondence to: Katsuhiro Toda, Department of Orthopedic Surgery, Kitahiroshima Town Toyohira Hospital, 4705, Azaka, Kita-Hiroshima Town, Yamagata-Gun, Hiroshima, 731-1222, Japan, Phone: +81-826-84-1155; Fax: +81-826-84-1015; E-mail: goutattack@yahoo.co.jp

Key words: pharmacological treatment, dilemma, pain, advantages, disadvantages Received: May 16, 2018; Accepted: May 21, 2018; Published: May 27, 2018 